\title{
Hereditary hemorrhagic telangiectasia: fatigue and dyspnea
}

\author{
Farzan Irani MD, Rahil Kasmani MD
}

$\infty$ See primer by Grand'Maison, page 833, case by Manawadu and colleagues, page 836, and clincial image by Nanda and Bhatt, page 838

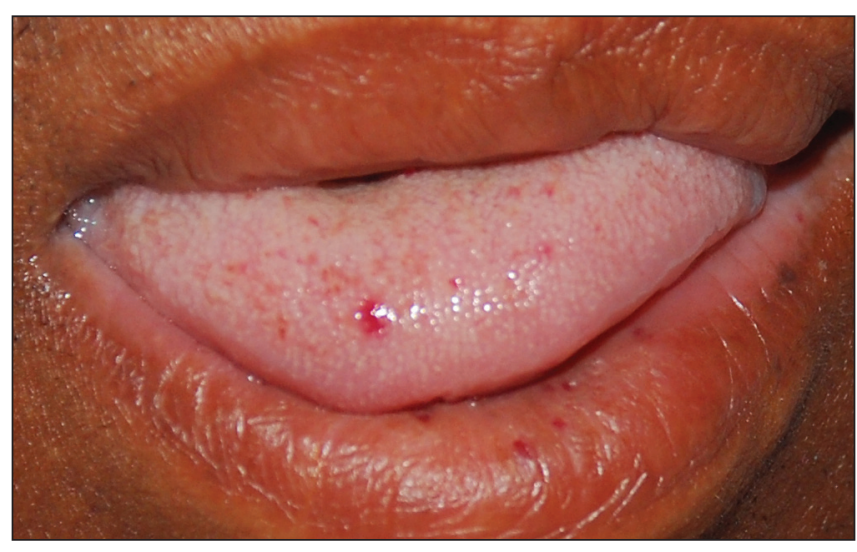

Figure 1: The patient had multiple telangiectasias on his tongue and lower lip.

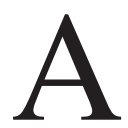

49-year-old man presented to the emergency department with a 2 -week history of progressive fatigue and dyspnea, as well as recurrent epistaxis. Laboratory investigations revealed severe hypochromic microcytic anemia, with a hemoglobin level of 48 (normal $120-140) \mathrm{g} / \mathrm{L}$. He reported that his mother and brothers also had recurrent nosebleeds.

We observed multiple telangiectasias on the patient's lower lip (Figure 1), tongue and palate. Nasal endoscopy showed active bilateral bleeding from the septum with crusting and erosions. No septal telangiectasias were found. Upper gastrointestinal endoscopy showed small nonbleeding antral and duodenal arteriovenous malformations (Figure 2). The results of a colonoscopic examination were normal.

A computed tomography (CT) scan of his chest showed multiple arteriovenous malformations, with the largest in the anterior left lower lobe. A CT scan of the patient's abdomen showed a large irregular arteriovenous malformation in the posterior segment of the right lobe of his liver (Figure 3), and a few other small malformations.

Hereditary hemorrhagic telangiectasia was diagnosed based on the patient's recurrent epistaxis, multiple mucocutaneous telangiectasias, visceral arteriovenous malformations and family history. ${ }^{1}$ Financial constraints prevented genetic testing. Because our patient did not report headaches or other neurological symptoms, we did not perform cerebral imaging.

Neither repeat endoscopy nor nasal surgery was performed, and his nosebleed was controlled with anterior nasal

From St. Vincent Mercy Medical Center, Toledo, USA

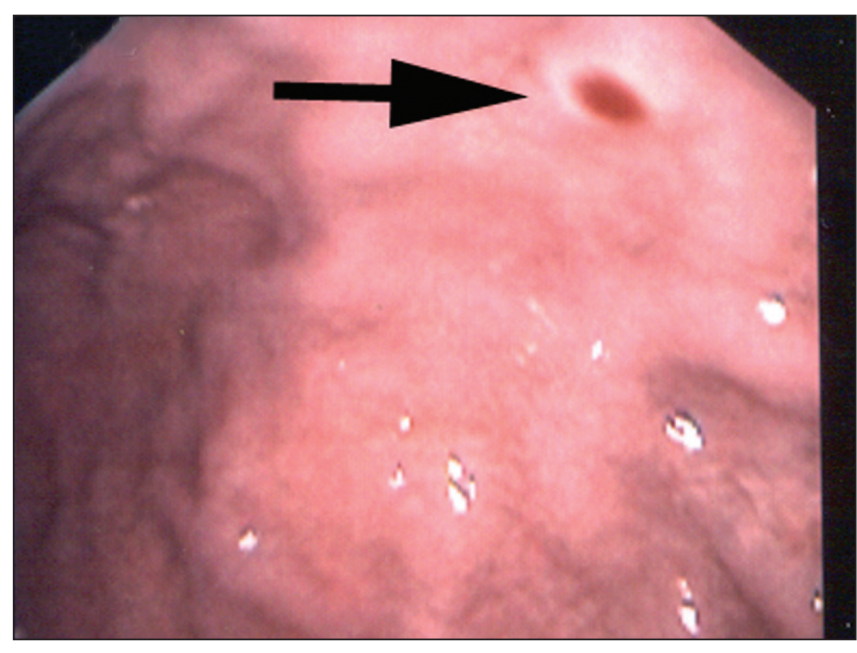

Figure 2: A gastric arteriovenous malformation surrounded by an anemic halo (arrow).

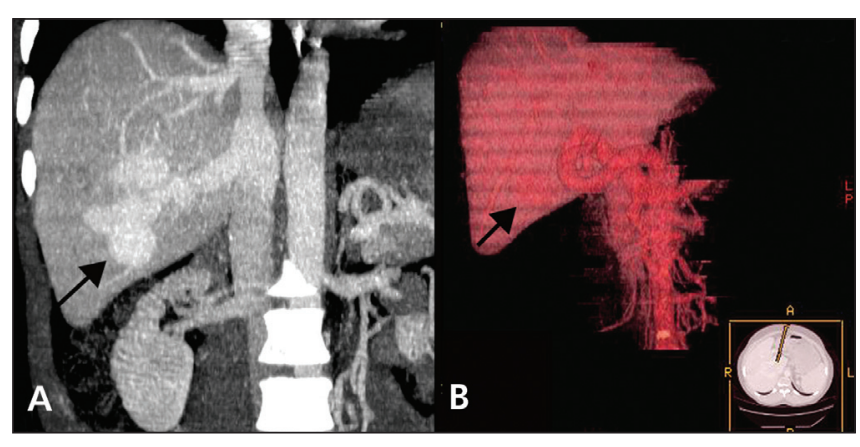

Figure 3: (A) A computed tomography scan of the patient's abdomen (coronal view) showing a large hepatic arteriovenous malformation (arrow). (B) A 3-dimensional reconstructed image of the patient's liver showing a large arteriovenous malformation (arrow).

packing only. A blood transfusion resulted in improvement of his symptoms. The patient's care was continued at an outpatient hematology clinic.

Acknowledgement: We thank Ms. Joyce Moses BSRT for her invaluable assistance in the 3-dimensional image reconstructions.

\section{REFERENCE}

1. Shovlin CL, Guttmacher AE, Buscarini E, et al. Diagnostic criteria for hereditary hemorrhagic telangiectasia (Rendu-Osler-Weber syndrome). Am J Med Genet 2000;91:66-7. 